\title{
VISUALIZATION METHODS FOR TIME-DEPENDENT DATA - AN OVERVIEW
}

\author{
Wolfgang Müller \\ Postfach 220 \\ PH Ludwigsburg \\ Dept. of Mathematics and Computer Science \\ D-71602 Ludwigsburg, GERMANY
}

Heidrun Schumann

\author{
University of Rostock \\ Dept. of Computer Science \\ D-18051 Rostock, GERMANY
}

\begin{abstract}
Visualization has been successfully applied to analyse time-dependent data for a long time now. Lately, a number of new approaches have been introduced, promising more effective graphs especially for large datasets and multiparameter data. In this paper, we give an overview on the visualization of time-series data and the available techniques. We provide a taxonomy and discuss general aspects of time-dependent data. After an overview on conventional techniques we discuss techniques for analysing time-dependent multivariate data sets in more detail. After this, we give an overview on dynamic presentation techniques and event-based visualization.
\end{abstract}

\section{MOTIVATION \& BACKGROUND}

The analysis of time-series data is one of the most widely appearing problems in science, engineering, and business. In the last years this problem gained increasing importance due to the fact that more sensitive sensors in science and engineering and the widespread use of computers in corporations have increased the amount of time-series data collected by many magnitudes.

For long, visualization has proven to be an effective approach to analyze time-series data. The motivation behind this approach is to exploit the phenomenal abilities of the human eye to detect structures in images. A well designed visualization can aid in answering the following questions for unknown temporal data (MacEachren 1995):

- Does a data element exist at a specific time? (Existence of a data element)

- When does a data element exist on time? Is there any cyclic behavior? (Temporal location)

- How long is the time span from beginning to end of the data element? (Temporal interval)
- How often does a data element occur? (Temporal texture)

- How fast is a data element changing or how much difference is there from data element to data element over time? (Rate of change)

- In what order do data elements appear? (Sequence)

- Do data elements exist together? (Synchronization)

The visualization of time-dependent data has a long history. Time-series plots appeared for the first time in the illustration of planetary orbits in a text from a monastery school in the $10^{\text {th }}$ or $11^{\text {th }}$ century (Tufte 1983). In science, time-series charts have been rediscovered not earlier than in the $18^{\text {th }}$ century by Lambert. He applied line graphs to display periodic variation in soil temperature in relation to depth under the surface (Lambert 1779). Today, a wider repertoire of techniques to visualize time-dependent data is available.

In this paper, we give an overview of different techniques suitable to analyze time-series data. In section 2 we introduce a taxonomy for time-dependence of data. In section 3 we give an overview on visualization techniques for this problem field. Our classification of visualization techniques distinguishes between static and dynamic representations as well as between more conventional techniques and special methods for multi-parameter data. Moreover, we discuss the common approach of event-based visualizations. We conclude in section 4 with general remarks on using the described techniques and issues for further research.

\section{TAXONOMY}

Before discussing visualization methods for timedependent data we want to clarify some basic notation. We consider multi-parameter data as a set of multidimensional 
multivariate data. We adopt the convention from Bergeron (Bergeron 1993) and Wong (Wong 1997) that the term multidimensional refers to the dimensionality of the independent variables, while the term multivariate refers to the dimensionality of the dependent variables of a data set. In our case the parameter time has an extraordinary importance, defining the independent dimension for which all other variables are given. That means, we consider multivariate data over time. Therefore, we can define that time dependent data is characterized by data elements being a function of time. In general, data takes the following form:

$$
d=f(t)
$$

For data defined at discrete time stamps $t_{i}$, this relation can be represented as:

$$
D=\left\{\left(t_{1}, d_{1}\right),\left(t_{2}, d_{2}\right), \ldots,\left(t_{n}, d_{n}\right)\right\}
$$

where

$$
d_{i}=f\left(t_{i}\right)
$$

The data elements $d_{i}$ can represent different data types. Usually we differentiate between nominal, ordinal, and quantitative data, or tuples of these, whether an inherent ordering, or a meaningful distance metric is given for the data or not. In the case of multivariate data, a data element $d_{i}$ combines at least the values of two variables.

Besides the data elements, the time axis itself can be characterized in more detail. For this purpose Frank introduced a taxonomy and distinguishes between (Frank 1998):

- Discrete time points vs. interval time:

Discrete time points describe the time as abstractions, comparable to discrete Euclidian points in space. These points have no duration. In contrast to that, interval time uses an interval scaled time axis like days, months, or years. In this case, data elements $d_{i}$ are defined for a time duration, specified by two time points.

- Linear time vs. cyclic time:

Linear time assumes a starting point and defines a linear time axis with data elements from past to future. However, many natural processes are cyclic like the cycle of the seasons. To meet these requirements a cyclic time axis can be used. The point order of a cyclic time axis is meaningless with respect to a cycle, e.g. morning is before evening, but the morning also comes after the evening.

- Ordinal time vs. continuous time:

These variants distinguish, whether the time axis is scaled ordinal or continuously. In the first case, only statements like "before" or "after" are possible. In the second case, we can quantify the time difference between the appearance of two data elements.

- Ordered time vs. branching time vs. time with multiple perspectives:
Ordered time axes consider events that happen one after the other. That means, data element $d_{i}$ follows $d_{i-1}$ in time and precedes $d_{i+1}$. Branching time is used for scenarios where sequences of actions are foreseen. In this case multiple alternatives are possible, depending on future decisions: Each data element $d_{i}$ can be followed by the data elements $d_{i+1}, d_{i+1}, d_{i+1}$ ', and so on. This kind of time axis supports decision making processes. Moreover, time with multiple perspectives allows specifying more than one data element $d_{i}$ for one time step $t_{i}$. In doing so, further elements $x_{i}, z_{i} \ldots$ can be associated with $t_{i}$ to define parallel events in time.

By using the taxonomy of Frank we have characterized the temporal aspects of the data. In the following chapter we will discuss how such data can be visualized.

\section{VISUALIZATION TECHNIQUES FOR TIME- DEPENDENT DATA}

The purpose of the visualization of time dependent data is to detect and validate characteristic properties of a unknown function $f$. In particular, we are interested in the exploration of time patterns, and the temporal behavior of data elements $d_{i}$ to answer the questions listed in section 1 .

For the visualization of time-dependent data we can distinguish two cases based on the time dependence of the visual representations:

- The visual representation is not time-dependent: The visual representation does not automatically change over time. Modifications result from user interaction only. In this case, we speak about static representations.

- The visual representation is time-dependent: The visual representation changes dynamically over time and is a function of time. We denote this form as dynamic representation.

Both forms have their specific benefits, and it has to be decided based on the concrete task, which kind of representation should be used. We cover static representations in section 3.1, and deal with dynamic representations in section 3.2 .

\subsection{Static Representations}

Static representations have been used to depict timedependence of data for quite a while now. This type of representation has certain benefits. Most important, they allow the conclusion of quantitative statements. Moreover, special data features and patterns as well as data values, time steps and positions can be explored according to the underlying scales without temporal limitations.

A number of well-known, conventional techniques have been developed in the past, and we will give a brief 
overview in the following section. Most of these techniques, however, are limited to the representation of a single variable over several time steps or a limited number of time variables and time steps. The visualization of multivariate data for longer time periods requires more effort, and is a current research topic. We discuss some novel approaches in section 3.1.2.

\subsubsection{Conventional visualization methods}

The ground for effective visualization has been prepared by Bertin (Bertin 1983), who distinguished 8 visual variables which could be used to encode nominal, ordinal or quantitative data visually. Mackinlay (Mackinlay 1996) carried on this work and presented an enhanced ranking of visual variables. All conventional visualization techniques can be understood as an application of the fundamental insights revealed by Bertin and Mackinlay.

A classical example for a very effective time-series chart is Minard's display of the fate of Napoleon's army on the campaign against Russia, presented almost 150 years ago (Minard 1844-70, see Figure 1). This graph is still be considered one of the best statistical charts ever drawn (Tufte 1983). It succeeds well in exploiting the spatial dimension and in applying additional visual variables to depict, the path of the campaign, the size of the army left, and the temperature (Elvins 1997).

More conventional approaches are based on the mapping of time on an available quantitative scale. In case of a 1D scale this leads to a Sequence Graph, in case of a 2D or 3D graph to a Time Series Graph. Like in standard cases, the appropriateness of a technique depends on the kind of data available, for instance Point Graphs for point data,

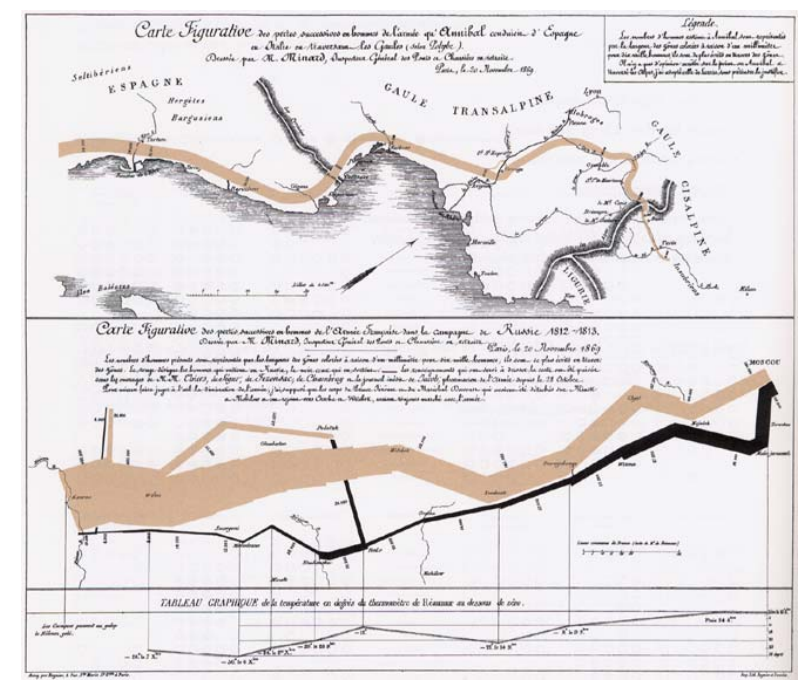

Figure 1: Minard's classical visualization of the fate of Napoleon's army on the campaign against Russia (Minard 1844-70, from Tufte 1983)

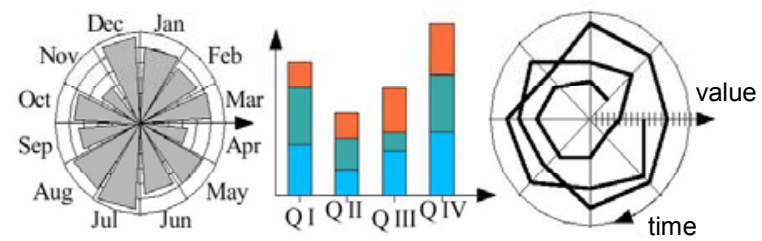

Figure 2: Graphs for time-dependent data: left: Sector Graph, middle: Stacked Bar Chart, right: Circle Graph (after Harris, 1996)

Line Graphs for continuous data, Bar Graphs for cumulative data, and Circle Graphs for cyclic data (Harris 1996; see Figure 2 for an example).

More complex graphs can be generated by mapping a static graph as an independent representation of the data element $d_{i}$ for a time-step $t_{i}$ onto a more general graph representation for more then one data element. In case the target graph is a sequence graph this leads to a Chess Plot (Monmonier 1990), in case of a time series plot to the concept of Composite Graph, or, in a quite modern interpretation using the third dimension, to a Worlds-within-Worlds visualization (Feiner 1990).

Even one step further are approaches which succeed in linking such independent representation of data for each time-step to a single map. Examples are the Change Chart, which presents an evolution of a symbol to display changes in data over time, and the Stacked Bar Chart, which represents the total amount of data as a single bar and depicts the increases at each time step by colour or texture. The Parallel Coordinates Technique (Inselberg 1997) somehow also belong into this category. Here, a number of rotated sequence charts are connected by linking corresponding data elements in each graph. Originally used to

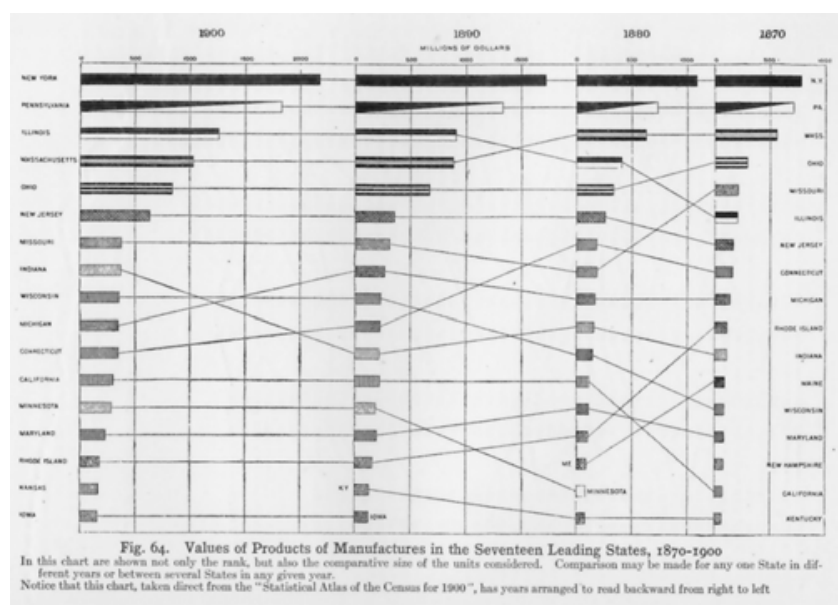

Figure 3: Parallel Coordinates for the visualization of time-dependent data (Garnett 1903, taken from Brinton 1919). Note the reverse arrangement of years from right to left. 


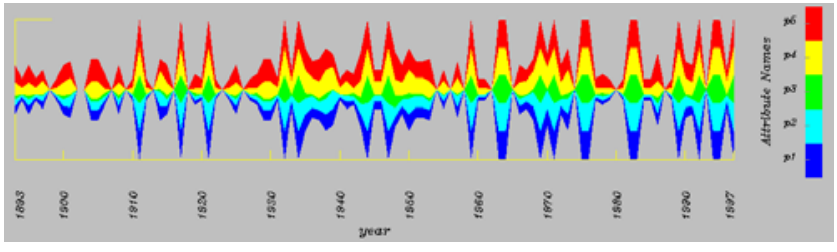

Figure 4: ThemeRiver visualization for 100 years of climate data.

visualize multivariate data by mapping on the different sequence charts, this type of plot may also be used to present time-series data by mapping the different time-steps to the individual axis. In this form, Parallel Coordinates have actually been used much longer (see Figure 3).

Last not least, interaction techniques have also been utilized to present temporal aspects. Most prominent is the Temporal Brushing, where the brushing of a timeline leads to the rendering of an appropriate graphical representation for the selected time-step, and Temporal Focusing, where a detailed but static view is presented for a selected time frame (Monmonier 1990).

Most of the techniques mentioned above have been designed with the focus on presenting simple forms of time-series data. Nowadays, the analysis of multivariate time-series data becomes more and more important. The next chapter gives an overview of a number of techniques specifically designed for this purpose.

\subsubsection{Visualization methods for multivariate data over time}

Visualizing multivariate data over long time periods requires special effort. In this case the data element $d_{i}$ for a given time step $t_{i}$ covers the data values for more than one variable. We present a selection of methods covering this topic.

A well-known technique dealing with this problem is the ThemeRiver (Havre 2000). This technique has been developed for document visualization: the frequency of the occurrence of special words in documents is counted for each time step. These occurrences are mapped to a special bar chart (with a continuous linear time axis). Interpolation between the bars (e.g. using Bezier splines) is used to generate the impression of a flowing surface for each word. The ThemeRiver technique allows an intuitive interpretation of temporal changes of document occurrences as well as their temporal relations.

Figure 4 shows an example of this technique representing the time series of normalized cluster centroids for 5 variables of a climate model data set. Low values or a thin river snapshot represent extremely cold summers, whereas high values or a broad river snapshot characterize extremely hot summers. Viewing this graph, the first impression is that the number of high values for most of the vari- ables representing the number of extreme hot summers increases with time in the second part of the 20th century. This is a special feature of the ThemeRiver technique, general trends are very well represented. However, parameters are not treated equally. Variables near to the middle axis have more weight, and parameters towards outer boundaries are represented with distortions. Due to this, interaction functionality for a parameter re-arrangement has to be provided allowing to put interesting parameters together and, in so doing, to investigate correlations of variables.

Another technique specifically designed to support the comparison and analysis of cyclic data is the Spiral Graph (Weber 2001). Here, the time axis is represented by a spiral. The data values $d_{i}$ are rendered relatively to this spiral as points, lines or bars, depending on the characteristic of the data. Hence, this technique is as flexible as standard time series graphs. However, periodic structures can be detected much more easily. Moreover, it is possible to compare a small number of different data sequences by integrating them into the Spiral Graph. Figure 5 depicts an example for a Spiral Graph visualization. The detection of cycles in data can be enhanced by adding data analysis tools to detect prominent frequencies. These frequencies can be utilized to aid the user in the interactive steering process, i.e. by providing presets for time length to be mapped onto a spiral revolution.

Another way to present time dependent data is to use special visual metaphors. One of such metaphor being particularly intuitive is the Calendar View (van Wijk 1999). This technique was introduced to present clusters of daily data values. Figure 6 gives an idea of this approach. The left view shows a calendar plot, in which the days are color-coded with respect to their affiliation to a certain cluster. The color scale of the clusters is presented on the right hand side of the figure. Now one can choose clusters, days or month of interest, and associated data values are drawn in the middle of the figure. Further interaction functions as the selection of a mediod (mean trend) or a median (typical day representing its cluster) modus are also included. In doing so, typical and non-typical clusters and

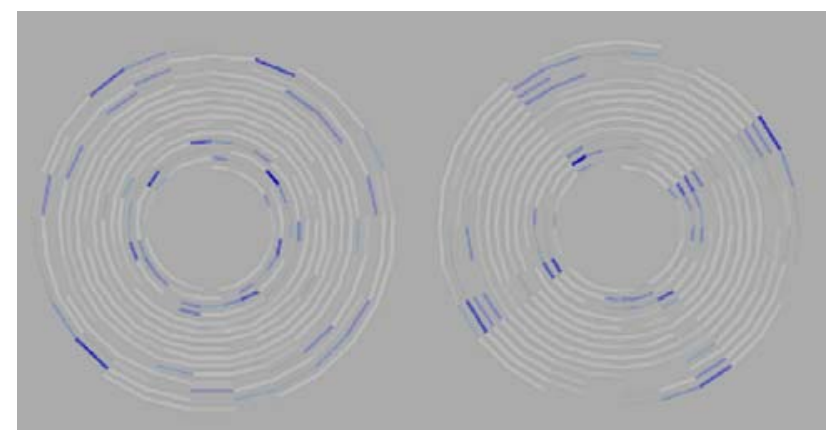

Figure 5: Spiral Graph visualization of monthly notifications of illness. Left: Assumed cycle of 27 days. Right: Valid cycle of 28 days. 


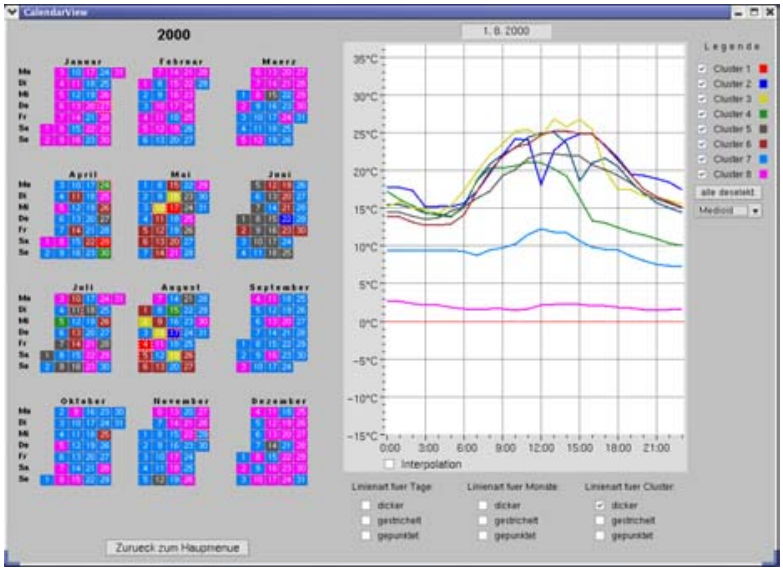

Figure 6: Calendar View based on (Wijk 1999): Cluster visualization of daily temperature cycles based on hourly measured observations at the station Potsdam during the year 2000 (using the correlation coefficient as similarity measure).

relations can be identified effectively.

Other examples for useful metaphors are a clock or a pencil. The technique SpiraClock uses a clock to present cyclic data (Dragicevic 2002). By moving the hand of the clock for- or backwards, data as well as special events are drawn or removed. If the amount of data to be presented exceeds a given threshold, the data of early time steps will be aggregated automatically. The technique Lexis Pencils (Brian 1997) maps different time-dependent variables onto different faces of a pencil, starting with the top of the pencil (see Figure 7). Advantages of this approach are, the combination of continuous and discrete variables in one plot, different pencils can be presented together, and pencils can be located in 3D space to show the spatial context of time-dependent data as well.

Beside using metaphors to generate clear and expressive displays common visualization techniques can be extended to time-dependent data. One example of such an approach is the technique Wormplots (Treinish 1997), which is based on the well-known Scatterplot. For each discrete time step $t_{i}$ a Scatterplot is drawn. Point clouds are identified in the first plot, and represented by little circles. The behavior of these point clouds is traced over time, and the associated circles are connected from one time step to the following by linear interpolation. In doing so, so-called worms are generated and displayed, which represent the temporal behavior of a point cloud. Figure 8 gives an example of this idea.

Another common visualization approach is axis-based visualization. In this case axes are drawn and scaled with regard to the range of associated variables. In section 3.1.1 we have already given some examples for that including traditional techniques using only one axis, or the technique of Parallel Coordinates using one axis for each variable of
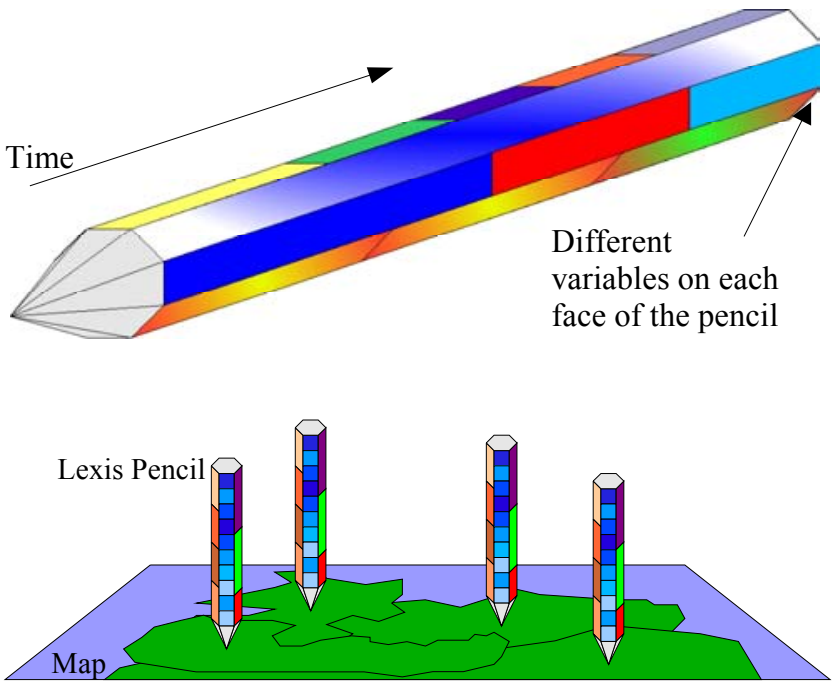

Figure 7: Example for a Lexis Pencil visualization.

a multivariate data set. Now we will further introduce 2 axis-based approaches, considering multivariate data as well as the extraordinary importance of the time axis (Tominski 2003). The TimeWheel technique presents the time axis in the center of the display, and arranges the other axes circularly around it (see Figure 9). A single colored line segment connects a time step on the time axis and corresponding data values on the variable axes. It is obvious, that the temporal behavior of a variable can be explored most efficiently, if the variable's axis is arranged parallel to the time axis. Otherwise, when an axis is almost

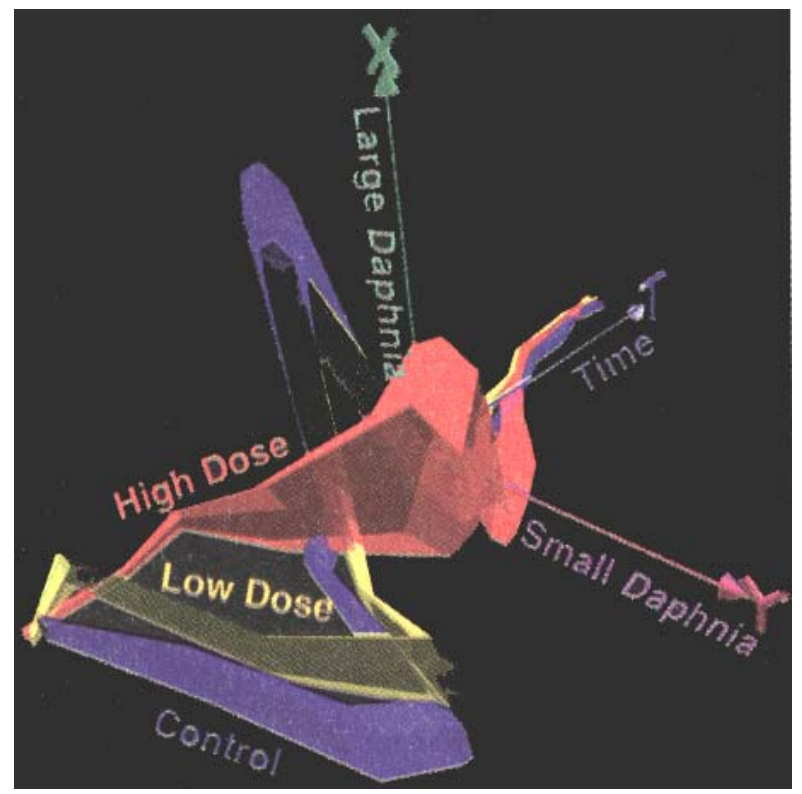

Figure 8: Wormplot (Treinish 1997) 


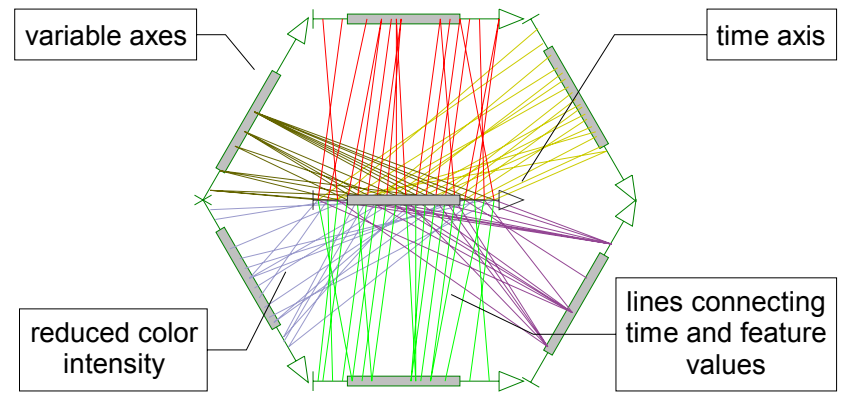

Figure 9: Demonstration of the TimeWheel technique:. 6 variable axes are arranged circularly around an exposed centered time axis.

perpendicular to the time axis its visual analysis is very difficult. Therefore, shortening of axis length and color fading is provided according to the angle with the time axis (see Figure 9). Interactive rotation of the TimeWheel is supported so that a user can move axes of interest into a position parallel to the time axis.

Another axis-based technique is the MultiComb. Here, multiple time axis (one for each variable) are circularly arranged either outward from the center of the display or star-shaped (see Figure 10). To avoid overlapping plots axes do not start at the center of display. The center area can be used to draw further information like a special glyph for value comparison or an aggregated view of "past" values.

If the range of the time axis is rather large, or many variables have to be considered, a visual presentation might be very complex. In such situations it can be useful, not to display data values directly, but to draw special events of interest based on the data values. We will discuss this approach in more detail in section 3.3.

\subsection{Dynamic Representations}

Except for the interaction techniques mentioned above, none of the discussed techniques has applied temporal aspects to represent data. Dynamic representations, on the other hand, make direct use of time to depict some data. They are able to demonstrate the general temporal behaviour, the dynamic of data and processes very well. Let the data elements $d_{i}$ be given over a time period from $t_{\text {start }}$ to $t_{\text {end }}$. The most natural approach is to map the temporal aspects of the data directly onto the time control of a dynamic representation, where the data elements' representation changes over time (e.g. size, shape, colour, texture, transformation). Here, we have to consider the different cases, how the time dependence of the data values $d_{i}$ can be given (see section 2).

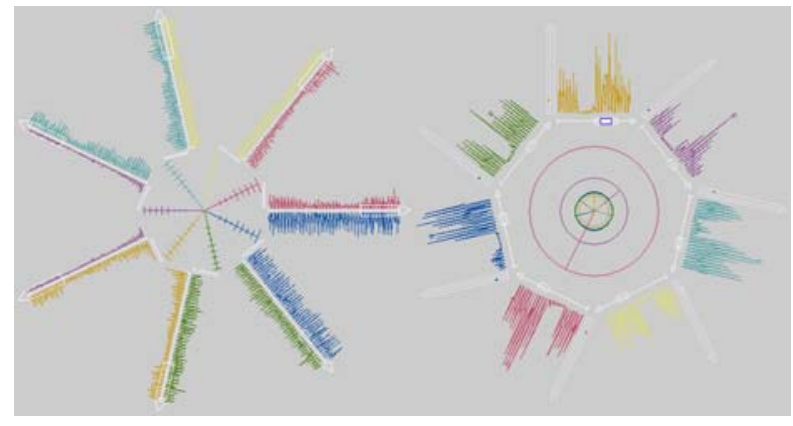

Figure 10: Two views of different data sets visualized with MultiCombs. On the left, time axes extend from the center; an aggregation view and mirrored plots are provided. The figure on the right shows variable axes extending from the center and circularly arranged time axis.

In case of continuous linear time it is a natural approach to represent the time $t$ found in the data directly and simply to generate appropriate representations for the data values $d$ depending on the time $t$. In fact, we have to sample $f(t)$ continuously regarding time to get data elements $d_{i}$ to be visualized. This leads to a computer animation, where each element $d_{i}$ corresponds to a frame $i$ in the animation.

To guarantee the perception of continuous movements and changes the rate of repetition of images must be high enough to guarantee smooth motion from frame to frame. Here, the sampling rate should not drop under 15 frames per second; continuous movements start being perceived smooth around 30 frames per second. If this sampling rate cannot be achieved, the animation displays jerkiness and quality usually drops dramatically. The reason for this is the sensibility of the human visual systems to sudden changes in the visual field, attracting out attention immediately. Lessons learned from video quality measurements show that in this case it might be a good approach to work without a continuous animation and to assume interval time instead, which leads to other approaches discussed below.

It is not always suitable to map the observed time interval $\left\langle t_{\text {start }}, t_{\text {end }}>\right.$ directly to the duration of the whole animation, e.g. to wait for days to see a change in the visualization of a weather simulation. In general, a positive scaling factor $s$ is used to adjust the duration of the animation appropriately, leading to

$$
t^{\prime}=s \cdot\left(t-t_{\text {start }}\right)
$$

where $t^{\prime}$ denotes the animation time. We can distinguish between

- Time-lapse visualizations $(s<1)$,

- Real-time visualization $(s=1)$, and

- Slow motion visualizations $(s>1)$.

In the exploration of the data in interactive visualization environments and for presentations it is also typical to vary 
$s$ in time to spend more time on interesting time spots while passing intervals with no apparent changes.

For interval data, the depiction of smooth movements for changes is not of interest. Nevertheless, time can be mapped to the temporal aspect of the presentation. In this case, this leads to a presentation similar to a slide show, where data elements $d_{i}$, are presented sequentially over time. The difference with respect to animation is that in this case no attempt is done to achieve the perception of a continuous movement. Again, experiences from corresponding video presentations have shown that display time of around and over 1-2 seconds for each frame seem to be appropriate. As mentioned before, continuous time data can be presented using the same metaphor. In this case, the result is a flashlight visualization, whereby data samples $d_{i}$ are interpreted as interval data.

For discrete time steps, we can actually take the same approach and present the data in terms of a flashlight visualization. More common, however, is to assume an underlying data model with continuous time. If time samples $t_{i}$ are regular and close enough to achieve the perception of continuous movements, we proceed as in the case of continuous data. Often, however, this will not be the case and data values will have to be interpolated based on local temporal neighbourhood. This technique is called inbetweening. Various interpolation methods have been proposed for this purpose. In general, the interpolation method has to suit the underlying data model. Moreover, if the interpolation display is not continuous, the resulting animation will again display disturbing jerkiness. Thus, linear interpolation is not appropriate in many cases. Special interpolation techniques have therefore been proposed to overcome this problem. For instance, in computer animation for sampling data based on moving physical bodies interpolation methods are commonly applied which give the impression of preservation of the moment of intertia, depicting slower movements at the begin and end of the trajectory (slow in, slow out; Lasseter 1987).

In case of static data we can nevertheless utilize time to enhance the visualization, either to extend the amount of data displayed, or to depict multivariate aspects of the data. We are also free to map ordered or quantitative attributes onto the free parameter time.

\subsection{Event-based Visualization}

The techniques discussed above assume generally an underlying linear discrete or continuous time model. A specific case is present when the data is event-based. Here we assume that an event represents an occurrence in time signaling a change in data. A general aspect of event-based data is that time between following events is usually not constant.

Existing event-based data can be visualized using the same approaches as for discrete or continuous time data.
The situation is however different when incoming events and the changes in data shall be visualized in real-time, e.g. for monitoring or controlling of a process. Since the occurrence of events is a priori not known, changes in the data cannot be foreseen. This puts restrictions on the application of certain visualization techniques, e.g. the interpolation of data values.

The applicability of general approaches from the field of information visualization especially in this field, has been proven e.g. by Matković et. al. (Matković 2002) who applied successfully level-of-detail and focus and context techniques for real-time monitoring of processes. For this, they introduce the concept of multi-instrument (e.g. MultiBar and Multi-Gauge) allowing to monitor several data elements at a time at lower level of detail and to access more detailed information by focusing.

Event-based visualization plays an important role in the field of simulation. Here, it not only allows to present the data for effective analysis but also to interact with the simulation in real-time: parameters can be adapted during the simulation and even the simulation model can be changed by adding or removing elements interactively. The user may also play an active role in the simulation, adding new events by its own. Examples for such applications can be found e.g. in online training situations in distributed simulators. VR techniques have gained increasing interest in such scenarios (see Figure 11 for an example).

Events represent the point in time when a change is about to happen with regard to the data and the underlying model. As such, these events are of special interest. Dynamic visualization is a valuable tool to detect these events and the corresponding changes in time-series data. However, especially in the cases of long-lasting simulations or multivariate and complex data it might still be difficult to detect important events and critical changes. Comparable to the detection of features in the spatial domain, corresponding techniques have lately been applied to also detect events in given data. Post et.al. apply this idea to the analysis of flow field data (Post 2002). Here, events are interpreted as the counterpart of spatial features in the evolution of features. In this context they distinguish between the following types of events on spatial features:

- Birth or death,

- Entry or exit

- Split or merge,

- Topology change, and

- Collision.

Post et.al. provide first approaches to detect and extract such events based on rules. The development of corresponding techniques and rules for other kinds of domains and data is still an open problem. 

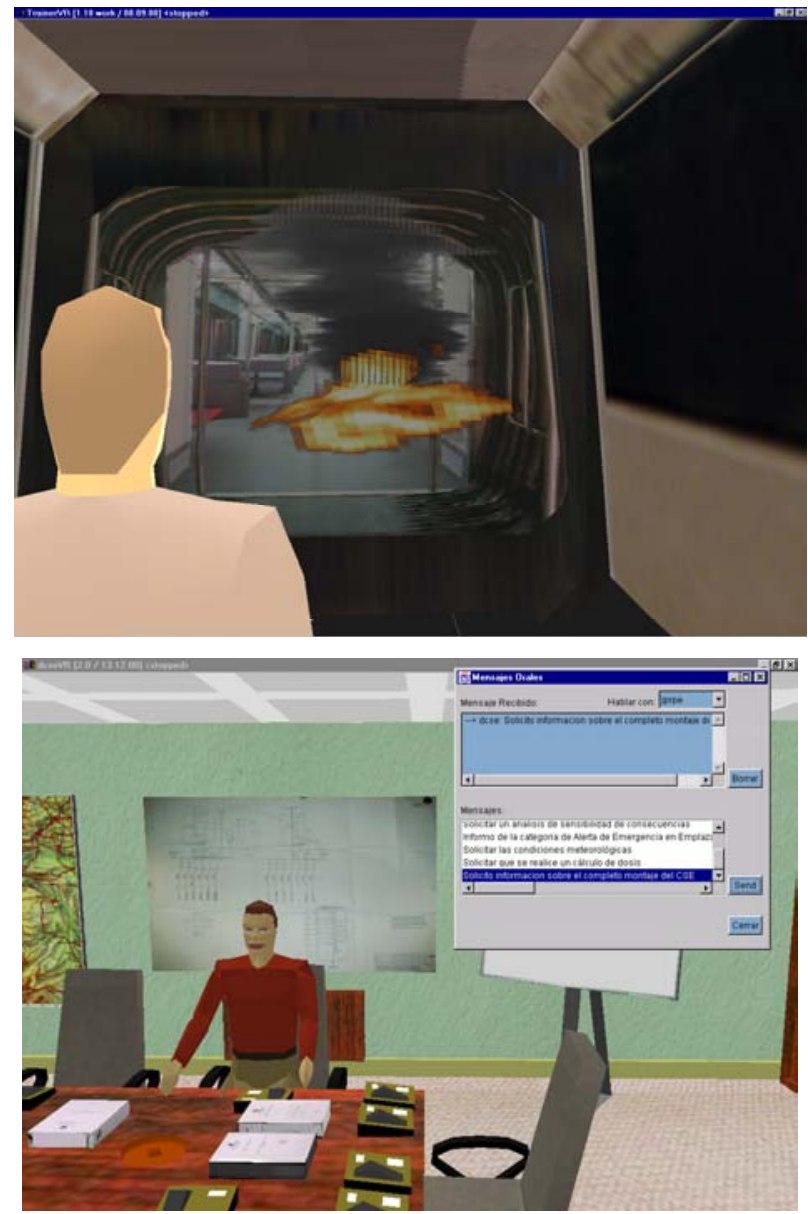

Figure 11: Event-based simulation and visualization. The images depict excerpts from an online emergency simulation for metro stations. Characters are controlled either by human trainees or software agents taking the role of an individual in the simulation (C) Fraunhofer AGC 2002, see Dörner 2000 for a more detailed discussion).

\section{CONCLUSION AND OUTLOOK}

The visual exploration of time-dependent data requires a special treatment of the factor time. This fact is often neglected, since common visualization systems consider the time as a parameter among others leaving the control about it's visualization in the hand of the user. On the other side, a number of special-purpose visualization techniques for time-dependent data have been developed lately. Each approach has specific characteristics and advantages. One has to decide, which technique to apply for a given problem. The following set of alternatives is a good starting point to find the appropriate technique:

- Static vs. dynamic representation.

Depending, whether one is interested in detailed exploration without time limitations, the correla- tive display of multivariate data, or in general trends and changes;

- Data vs. event visualization.

Depending on the amount of a data set, or the interest in special events;

- Conventional vs. multivariate display.

Depending, whether the temporal behavior of only few variables or the analysis of multivariate correlations is of special interest.

Since there is more then one technique available for each alternative, further decisions have to be made based on the characteristics of the time-dependence (e.g. cyclic or linear, see section 2).

Considering these aspects, visualization can surely support the exploration of time-dependent data. Further research seems however to be necessary to couple the presented visualization methods with those depicting also the spatial context, since many time-dependent data sets exhibit spatial dependencies as well. Here, only first approaches are available. Moreover, in the field of eventbased visualizations scientific work just has started, and further concepts must be developed.

\section{ACKNOWLEDGMENTS}

We would like to thank Christian Tominski and Thomas Nocke for valuable discussions and their support in the implementation of several of the presented techniques. Last not least, we would like to thank Rene Rosenbaum for proofreading.

\section{REFERENCES}

Bertin, J. Semiology of Graphics. The University of Wisconsin Press, USA.

Brian, F., and J. Pritchard. 1997. Visualisation of historical events using Lexis pencils. Centre for Applied Statistics Fylde College, Lancaster University, USA.

Brinton, W.C. 1919. Graphic Methods For Presenting Facts, The Engineering Magazine Company, New York, USA.

Dörner, R., P. Grimm, and Ch. Seiler. 2000. Agents and Virtual Environments for Communication and Decision Training for Emergencies. (ed) C. Sierra et. al., Proc. of the Fourth International Conference on Autonomous Agents, ACM Press, New York, 50-51.

Elvins, T.T. 1997. VisFiles - Presentation Techniques for Time-series Data. Computer Graphics, 31(2): 14-16.

Feiner, St., and C. Beshers. 1990. World within Worlds Metaphors for Exploring n-Dimensional Virtual Worlds. Proc. ACM Symposium on User Interface Software and Technology (UIST '90), 76-83.

Frank, A.U. 1998. Different Types of "Times" in GIS. In Spatial and Temporal Reasoning in Geographic Information Systems. (ed) M.J. Egenhofer, R.G. Golledge. 
Oxford University Press, New York, Oxford, 1998, 40-62.

Garnett, H. 1903. Statistical Atlas - Twelfth census of the United States, taken in the year 1900. United States Census office, Washington.

Harris, R.L.. 1996. Information Graphics - A Comprehensive Illustrated Reference. Management Graphics, Atlanta, Georgia, USA.

Havre, S., B. Hetzler, and L. Nowell. 2000. ThemeRiver: Visualizing Theme Changes over Time. In Proc. IEEE Symposium on Information Visualization 2000 (InfoVis '00). IEEE Computer Society, Los Alamitos, USA, 115-123.

Inselberg, A. 1997. Multidimensional Detective, In Proc. IEEE Symposium on Information Visualization 1997 (InfoVis '97). 100-107.

Lambert, J.H. 1779. Pyrometrie. Berlin, Germany.

Lasseter, J. 1987. Principles of Traditional Animation Applied to 3D Computer Animation. ACM Computer Graphics, Volume 21(4):34-44.

MacEachren, A.M. 1995. How Maps Work, The Guilford Press, New York, USA.

Mackinlay, J. 1986. Automating the Design of Graphical Presentations of Relational Information. ACM Transactions on Graphics. 5 (2): 110-141.

Matcović, K., H. Hauser, R. Sainitzer, M.E. Gröller. 2002. Process Visualization with Level of Detail. Proc. IEEE Symposium on Information Visualization (InfoVis '02), 67-70.

Minard, C. J. 1844-1870. Tableaux graphiques et cartes figuratives. (Collection of graphic works) École Nationale des Ponts et Chausées (ENPC). Fol 10975.

Monmonier, M. 1990. Strategies for the visualization of geographic time-series data. Cartographica, 27(1): 3045.

Post F.H., H. Hauser, F.H., B. Vrolijk, R. Laramee, and H. Doleisch. 2002. Feature Extraction and Visualization of Flow Fields. Eurographics 2002 State of the Art Reports, The Eurographics Association, Saarbrücken, 69-100.

Schumann, H., and W. Müller. 2000. Visualisierung Grundlagen und allgemeine Methoden. SpringerVerlag, Berlin, 2000. in German.

Tominski, Ch., J. Abello, and H. Schumann. 2003. Axesbased Visualizations. Submitted to IEEE Symposium on Information Visualization 2003, (InfoVis '03), Seattle, USA.

Tominski, Ch., P. Schulze-Wollgast, and H. Schumann,. 2003. Visualisierung zeitlicher Verläufe auf geografischen Karten.; Proc. GeoVis'2003, Hannover, 2003, 47-57, in German.

Treinish, L., and D. Silver. 1997. Worm Plots. IEEE Computer Graphics and Applications, 17: 17-20.
Tufte, E.R. 1983. The Visual Display of Quantitative Information. Graphics Press, Cheshire, Connecticut, USA.

van Wijk, J.J., and E. van Selow. 1999. Cluster and Calendar-based Visualization of Time Series Data. In Proc. IEEE Symposium on Information Visualization (InfoVis '99) (ed) G. Wills, D. Keim. IEEE Computer Society, 4-9.

Weber, M., M. Alexa, and W. Müller. 2001. Visualizing Time-Series on Spirals. Proc. IEEE Symposium on Information Visualization 2001 (InfoVis '01), San Diego, USA, 7-13.

\section{AUTHOR BIOGRAPHIES}

WOLFGANG MÜLLER is a temporary Professor of Mathematics and Computer Science at PH Ludwigsburg, Germany. In addition to Visualization and Information Visualization, his research focuses on Multimedia User Interfaces and Agent-based applications. His research is motivated by problems in data mining and knowledge management. He can be contacted by e-mail at <mueller_wolfgang@ph-ludwigsburg.de>

HEIDRUN SCHUMANN is Associate Professor for Computer Graphics at the University of Rostock, Germany. Her research profile covers visualization techniques for high-dimensional data, visual data mining, visual interfaces for mobile handhelds, rendering, and image transmission and display. She was heading the cross- institutional research group ,MoVi - Visualization of Multimedia Information on Mobile Computer Systems", supported by the German Research Society (DFG). Current research projects focus e.g. on the visualization of climate and human health data as well as on problems of user defined image transmission. She can be contacted by e-mail at <schumanneinformatik.uni-rostock.de> 